\title{
Evolución de las características epidemiológicas y clínicas de pacientes adultos del programa nacional al inicio de la terapia anti-retroviral en la Cohorte Chilena de SIDA, 2001-2015
}

\author{
Carlos Beltrán, Pedro Zitko, Marcelo Wolff, Fernando Bernal, Alicia Asenjo, \\ Ana M. Fernández, Ana Miles, Elizabeth Barthel y Gonzalo Wilson \\ Co-autores Corporación Grupo SIDA Chile*
}

Corporación Grupo SIDA Chile (CB, MW, FB, AA, AMF, AM,

$E B, G W)$.

Unidad de Estudios Asistenciales Complejo Asistencial Barros Luco (PZ).

Financiamiento: Conflictos de interés:

Recibido: 25 de agosto de 2016 Aceptado: 1 de septiembre de 2016

Correspondencia a: Carlos J. Beltrán Buendía cabel@vtr.net

\section{Evolution of epidemiological and clinical characteristics of adults patients belonging to the national program at start of antiretroviral therapy in the Chilean AIDS Cohort, 2001-2015}

Background: Chilean AIDS Cohort is the oldest and extensive in Latin America and one of most numerous and with longer follow up time to international level. Records information from 14,873 patients out of approximately 22,000 in antiretroviral therapy in the public system and its results have allowed to know the national reality and have contributed to the adoption of public policies. Aim: To describe the demographic, clinical and immunological characteristics of patients who have started ART in Chile and its evolution over the past 15 years. Patients and Methods: The cases were stratified by five-year periods: 2001-2005, 2006-2010 and 2011-2015. The data analysis included calculating proportions, their respective confidence intervals $95 \%$ and $\chi^{2}$ test for significance analysis was applied. Results: $17.4 \%$ of patients starting ART are women and the proportion has remained relatively constant. The highest proportion of new HIV cases are 30 and 39 years old, nevertheless the layer of 15-29 years demonstrates a significant increase from 21.7 to $36.4 \%$ in $2011-2015$ especially in men. $12.1 \%$ of new cases are older than 50 years old with a stable trend over time; however, women over 50 have increased from 11.0 to $15.6 \%$. Antiretroviral therapy initiation with CD4+ T lymphocytes less than 200 cells $/ \mathrm{mm}^{3}$ has decreased from 79.7 to $42.4 \%$ and in stage $\mathrm{C}$ from 45.4 to $22.6 \%$. Late presentation to antiretroviral therapy is higher in men but this gap has narrowed in the last five years. Pneumocystis jiroveci, wasting syndrome, tuberculosis, Kaposi's sarcoma and esophageal candidiasis are the most common opportunistic diseases without significant changes in the three-year periods analyzed. In the last five years, $15.5 \%$ of opportunistic diseases occurs in patients with CD4+ TL > 200 cells/mm3. Discussion: Despite the limitations of observational studies present report describes the characteristics and evolution of the epidemics in Chile in the last 15 years. The infection occurs at younger ages in men, whereas in women there is an increase over 50 years old. Despite advances in treatment access have reduced late presentation to therapy, important challenges remain to achieve more timely initiation of antiretroviral therapy in accordance with WHO 90-90-90 goals.

Key words: Chilean AIDS Cohort; Latin America; HIV; young adults; adolescents; aging; late presentation; opportinistic infections; 90-90-90.

Palabras clave: Cohorte chilena de SIDA; Latinoamérica; VIH; jóvenes; adolescentes; envejecimiento; presentación tardía; infecciones oportunistas; 90-90-90.

*Coautores Corporación Grupo SIDA Chile: Afani A, Aguilar C, Alfaro R, Allendes G, Alejandro A, Arancibia J, Arredondo S, Arredondo R, Álvarez C, Amaro A, Amigo M, Aylwin M, Ayala M, Ajraz T, Alarcón T, Acevedo W, Arriagada M, Avilés I, Bahamondes L, Ballesteros J, Bastías C, Blackburn E, Blamey R, Blanco S, Bugueño R, Barrios M, Cabrera J, Cabrera A, Calvo M, Campillay R, Carreño V, Caro C, Caro I, Carrasco A, Carvajal E, Castelli A, Castillo Y, Cataldo M, Ceballos C, Chacón M, Chahín C, Chanqueo L, Cid A, Concha C, Contreras P, Cortés C, Cruz R, Daube E, De Andraca M, Delama I, Díaz V, Díaz R, Díaz S, Díaz H, Escobar V, Esquivel N, Ewert F, Ferrer P, Figueroa L, Fuentealba H, Fuenzalida M, Fuenzalida F, Gacitúa D, Gaete P, Gajardo K, Gallardo K, Gallo C, Gálvez R, García E, Gavrilovics A, Giadalah M, Goecke B, González A, González G, González A, González F, Greppi D, Guzmán G, Hernández S, Herrera P, Herrera M, Iglesias M, Irsula A, Iturra C, Jensen W, Jorquera V, Lagos M, Lasso M, Leyton E, López O, Marambio V, Mardones J, Marincovich B, Martínez M, Matamala D, Maturana P, Medina L, Miles A, Molina C, Moraga A, Moreno A, Muñoz M, Muñoz R, Muñoz M, Muñoz R, Muñoz K, Navarrete M, Navarro L, Northland R, Ocampo R, Oñat M, Orellana L, Palma V, Palma S, Paredes A, Pérez S, Pérez C, Pérez A, Pino P, Retamal A, Rodríguez $K$, Rodríguez $L$, Rodríguez $C$, Rojas $A$, Rojas L, Ross E, Rubilar E, Rusque A, Salinas V, Salvador F, Sánchez A, Sánchez M, Sciaraffia A, Sepúlveda M, Serri M, Sierralta G, Silva M, Silva M, Sobarzo J, Solís T, Soto J, Stipicic M, Suazo S, Sutar C, Tagle M, Tapia J, Tordecilla R, Toro C, Torres A, Ulloa K, Usedo P, Valdebenito S, Valenzuela L, Valenzuela M, Valladares C, Vásquez D, Vásquez P, Vega R, Vega C, Vergara M, Vidal C, Villarroel C, Yanine D, Zenteno M, Zuñiga K. 


\section{Introducción}

Las distintas metodologías de investigación científica han sido complementarias en el estudio de la pandemia de infección por VIH. Gran parte del conocimiento científico actual y de la evidencia que respalda las intervenciones preventivas y terapéuticas en la infección por VIH y su impacto poblacional proviene de estudios observacionales a gran escala, que son los análisis de cohorte. Este tipo de estudios permite abordar objetivos amplios, por períodos prolongados de seguimiento y evaluar resultados de la aplicación de programas de intervención en "el mundo real"'. Los principales estudios de cohorte provienen de Europa y Estados Unidos de América, con reportes de 10.000 o más pacientes en seguimiento desde antes del año 2000, tales como la cohorte Suiza, la "ATHENA Cohort" de Países Bajos, la cohorte Aquitaine en Francia y la ICONA en Italia y, especialmente a través de agrupaciones de cohortes como EuroSIDA, NA-Accord o ART-CC, con un gran número de pacientes ${ }^{2-4}$. Estos estudios han entregado información relevante sobre eficacia y mortalidad a largo plazo, factores bio-demográficos asociados a efectividad y toxicidad, incidencia de enfermedades marcadoras y no definitorias de SIDA, recuperación inmunológica y toxicidades específicas, entre otras. En el último tiempo se han comenzado a comunicar resultados, en general a menor escala, de cohortes de países en vías de desarrollo, especialmente asiáticos y africanos.

\section{Cohorte chilena de SIDA}

Chile posee un programa público ministerial centralizado de acceso ampliado a terapia anti-retroviral (TARV) y monitoreo a través de exámenes de carga viral (CV) y de recuento de linfocitos T CD4+ (LT CD4) en 38 centros de atención de adultos en el país con infección por $\mathrm{VIH}$, en base a guías clínicas nacionales que establecen criterios uniformes de manejo de la infección por VIH/SIDA. Este programa de acceso ampliado se inició en 2001 en el sistema público, alcanzando $100 \%$ de cobertura hacia el año 2003, con lo que Chile fue uno de los primeros países de la región en alcanzar este objetivo. Desde el inicio del programa el año 2001, el Grupo SIDA Chile, formado por los profesionales tratantes de los centros de atención de adultos con infección por VIH del sistema público de salud, comenzó una evaluación prospectiva y multicéntrica del impacto poblacional del acceso ampliado a TARV, con un estudio de cohorte de la totalidad de los pacientes atendidos en el sistema público de salud, la Cohorte Chilena de SIDA, la más antigua y extensa de la región y una de las más numerosas y con mayor tiempo de seguimiento a nivel internacional. Los diferentes resultados de este estudio han sido presentados y publicados en congresos y revistas científicas nacionales e internacionales y han permitido la adopción de políticas públicas basadas en la realidad nacional $^{5,6}$. El estudio de cohorte incluye, a la fecha, la información bio-demográfica, clínica, inmunológica y virológica de 14.873 pacientes de los aproximadamente 22.000 adultos en TARV en el sistema público, que atiende a $85 \%$ de las personas diagnosticadas con infección por VIH. Considera además, sus principales desenlaces, con un seguimiento promedio mayor a 5 años.

El objetivo de este estudio es describir las características demográficas, clínicas e inmunológicas de los pacientes adultos que han comenzado TARV en Chile en el sistema público de salud y su evolución en los últimos 15 años, en particular la distribución por sexo y edad, la prevalencia de enfermedades oportunistas y la presentación tardía a TARV.

\section{Pacientes y Métodos}

La base de datos de la Cohorte Chilena de SIDA dispone de información de pacientes ingresados a TARV desde 2001, acumulando 15 años de observación hasta 2015. Esta información incluye variables de ingreso (sexo, edad, etapa clínica e inmunológica, enfermedades oportunistas, escolaridad, etnia, co-infecciones, factores de riesgo cardiovascular, etc.), resultados finales (muerte, abandono), resultados intermedios (recuento de LT CD4, $\mathrm{CV}$, reporte de efectos adversos, fármacos de la TARV y cambios de éstos, etc.), entre otras.

La recolección de datos de los pacientes que inician TARV en los 38 centros del sistema público es efectuada por los equipos de salud de los centros e incorporada en forma remota a la base de datos. La recolección de datos se realiza en $100 \%$ del marco muestral (beneficiarios del sistema público de salud) por lo que sus resultados son representativos del país completo (sistema público). Los datos son revisados manualmente y analizados por el equipo de trabajo de la Cohorte Chilena de SIDA para la detección de discrepancias y son validados mediante consulta electrónica o telefónica con los centros de atención. En la instancia de análisis, los datos extremos ("outliers") son nuevamente validados mediante consulta a los centros, al igual que otras discrepancias que se puedan detectar.

Las variables demográficas como edad y sexo fueron recolectadas de manera consistente con la fecha de inicio de TARV. Las edades fueron categorizadas en tramos definidos. Los datos de LT CD4 y CV registrados corresponden a la medición previa más cercana al inicio de TARV, al igual que la etapificación según clasificación del CDC. Los niveles de LT CD4 fueron estratificados en tres categorías: $<200,200-499$ y $>499$ céls $/ \mathrm{mm}^{3}$, mientras que la CV fue estratificada dicotómicamente utilizando como punto de corte 100.000 copias ARN/mL. Concordante con lo anterior, las enfermedades oportunistas fueron extraídas del reporte realizado concomitante al inicio de TARV. 
Para determinar la presencia de co-morbilidades como hipertensión arterial, diabetes mellitus y dislipidemia, también se utilizó el registro más cercano a la fecha de TARV.

Dado que el llenado de la información se realiza en forma diferida de la atención clínica, la base de datos al momento de su cierre (31 de diciembre de 2015) contó sólo con cerca de $70 \%$ del total de pacientes en TARV en el sistema público chileno, faltando ingresar la información de los casos de más reciente incorporación.

\section{Análisis estadístico}

En este estudio, se obtuvieron desde la base de datos las principales características demográficas y clínicas de los pacientes que inician TARV en Chile y los casos fueron estratificados por quinquenios: 2001-2005, 2006-2010 y 2011-2015. El análisis de los datos incluyó el cálculo de proporciones señalando la distribución de la población por cada estrato de la variable de interés según quinquenio. En la mayoría de los casos, las proporciones son presentadas con sus respectivos intervalos de confianza $95 \%$. Cuando esto no sucede, se entregan los totales marginales que permiten calcular el respectivo intervalo utilizando la formula $+/-1,96^{*}\left(p^{*}(1-p) / N\right)^{1 / 2}$, donde "p" corresponde a la proporción y $\mathrm{N}$ al total de casos. Cuando fue necesario realizar un test de hipótesis se utilizó $\chi^{2}$. Los resultados son presentados utilizando mayoritariamente gráficos.

\begin{tabular}{|c|c|c|c|c|}
\hline \multirow[b]{2}{*}{ Región } & \multicolumn{3}{|c|}{ Quinquenio } & \multirow[b]{2}{*}{ Total } \\
\hline & 2001-2005 & 2006-2010 & 2011-2015 & \\
\hline I Región de Tarapacá & 91 & 149 & 169 & 409 \\
\hline II Región de Antofagasta & 154 & 140 & 2 & 296 \\
\hline III Región de Atacama & 65 & 61 & 2 & 128 \\
\hline IV Región de Coquimbo & 141 & 115 & 1 & 257 \\
\hline V Región de Valparaíso & 637 & 733 & 772 & 2.142 \\
\hline XIII Región Metropolitana de Santiago & 3.587 & 3.024 & 2.325 & 8.936 \\
\hline VI Región de O’Higgins & 132 & 114 & 2 & 248 \\
\hline VII Región del Maule & 124 & 149 & 3 & 276 \\
\hline VIII Región del Biobío & 289 & 356 & 3 & 648 \\
\hline IX Región de La Araucanía & 160 & 206 & 251 & 617 \\
\hline X Región de Los Lagos & 102 & 219 & 9 & 330 \\
\hline XI Región de Aysén & 10 & 6 & 0 & 16 \\
\hline XII Región de Magallanes y Antártica Chilena & 25 & 20 & 0 & 45 \\
\hline XIV Región de Los Ríos & 52 & 124 & 4 & 180 \\
\hline \multirow[t]{2}{*}{ XV Región de Arica y Parinacota } & 99 & 140 & 106 & 345 \\
\hline & 5.668 & 5.556 & 3.649 & 14.873 \\
\hline
\end{tabular}

\section{Aspectos éticos}

El estudio de cohorte fue revisado por la Comisión de Ética del Ministerio de Salud y autorizado, por cuanto no contiene información que vulnere la privacidad de las personas con la utilización de un código que resguarda la identidad.

Además, el estudio se enmarca en la obtención de datos epidemiológicos relevantes para la definición de políticas públicas que han sido puestos a disposición de la autoridad sanitaria del país. Los aspectos operacionales del estudio han sido financiados por la Corporación SIDA Chile, personalidad jurídica del Grupo SIDA Chile, que recibe financiamiento público y privado.

\section{Resultados}

La distribución de casos según región para cada quinquenio explorado se presenta en la Tabla 1, con un total de 5.668 inicios de TARV en el quinquenio 2001-2005, 5.556 en el quinquenio 2006-2010 y 3.649 en los últimos cinco años de este reporte. La Región Metropolitana representa más de la mitad de los casos en todos los quinquenios (60,1\% en total). Es posible observar un importante aumento de casos entre los períodos 20012005 y 2006-2010 en las regiones de Los Ríos (138\%) y de Los Lagos (115\%) aun cuando debe considerarse que los datos del período 2011-2015 son aún parciales. En contraste, las regiones Metropolitana, de Valparaíso y de Coquimbo presentan disminuciones cercanas a 15\%. El resto de las regiones mantuvo su número de casos o bien los aumentó levemente.

\section{Distribución por sexo y edad}

En la Cohorte Chilena de SIDA, 17,4\% de los casos que inician TARV corresponde a mujeres y la relación mujer/hombre se ha mantenido relativamente constante con una proporción de mujeres de 17,1\% (2001-2005), $19,3 \%$ (2006-2010) y 15,2\% (2011-2015).

En el análisis de distribución por edad, destaca el grupo de adultos entre 30 y 39 años, aun cuando en el último quinquenio se ve superado por el estrato de 1529 años (Figura 1). Este último grupo de adolescentes y adultos jóvenes evidencia un importante aumento desde 21,7\% en 2001-2005 a 36,4\% en 2011-2015 (Figura 1). Al analizar este tramo de edad estratificado en rangos de edad más pequeños, se aprecia que este incremento ocurre principalmente entre los 15 y 19 y entre los 20 y 24 años de edad. Estos tramos evidencian una clara tendencia al alza, con incrementos relativos de 62,8 y $33,1 \%$, respectivamente. No obstante, el rango de 25 a 29 años continúa siendo el más importante en el grupo etario 15-29 años aunque su proporción presenta una disminución estadísticamente significativa (valor 


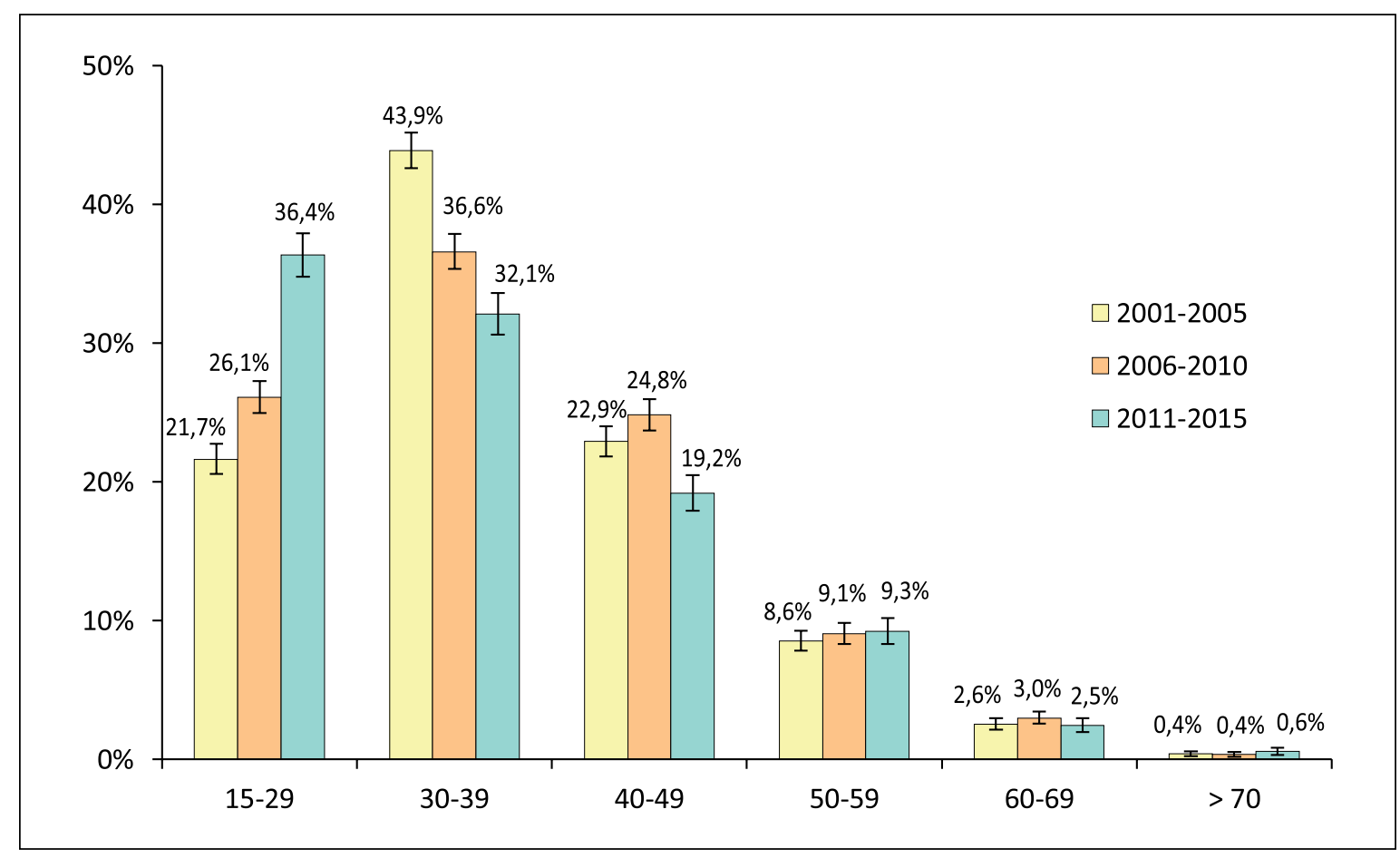

Figura 1. Distribución de edad de casos que inician TARV de la Cohorte Chilena, por quinquenio. Total de casos quinquenios 2001-2005: 5.658; quinquenio 2006-2010: 5.556; quinquenio 2011-2015: 3.649. En las barras se ilustran intervalos de confianza $95 \%$. $\mathrm{p}<0,05$; Figura 2). El aumento en el número de casos en la población adolescente y adultos jóvenes se observa especialmente en hombres en el grupo 15-29 años con un aumento en su representación desde 20,0 a $37,4 \%$ en desmedro de los grupos 30-39 y 40-49 años, que disminuyen su representación de 44,9 a $32,1 \%$ y de 23,5 a $18,7 \%$, respectivamente (Figura $3 a$ ), al inicio de TARV. En cambio, en mujeres se observa que los grupos de 15-29 años y 40-49 años se mantienen estables en el tiempo, observándose también una disminución del grupo de 30-39 años desde 39,0 a 32,0\% (Figura 3b).

Otro grupo etario relevante de observar corresponde al de pacientes mayores a 50 años de edad que ingresan a TARV. Este segmento representa $12,1 \%$ del total de los inicios de TARV con una tendencia estable en el tiempo (Figura 1). Sin embargo, en el análisis diferenciado por sexo se observa que esta tendencia estable corresponde a hombres, con 11,6 y 11,7\% de mayores de 50 años en el primer y último quinquenio, respectivamente (Figura 3a). En cambio, se observa un aumento sostenido, estadísticamente significativo, en la proporción de mujeres mayores de 50 años que inician TARV, desde 11,0 a 15,6\% para los mismos quinquenios (Figura 3b). Estas diferencias entre hombres y mujeres se expresan principalmente en el tramo de 50-59 años.

Entre los pacientes mayores de 50 años, $10,0 \%$ presenta hipertensión arterial, 9,2\% diabetes mellitus y
$5,0 \%$ dislipidemia. En este grupo etario, $21,8 \%$ presenta al menos una de estas patologías constitutivas de riesgo cardiovascular antes del inicio de TARV, contra sólo 9,5\% entre los pacientes bajo 50 años de edad (datos no mostrados).

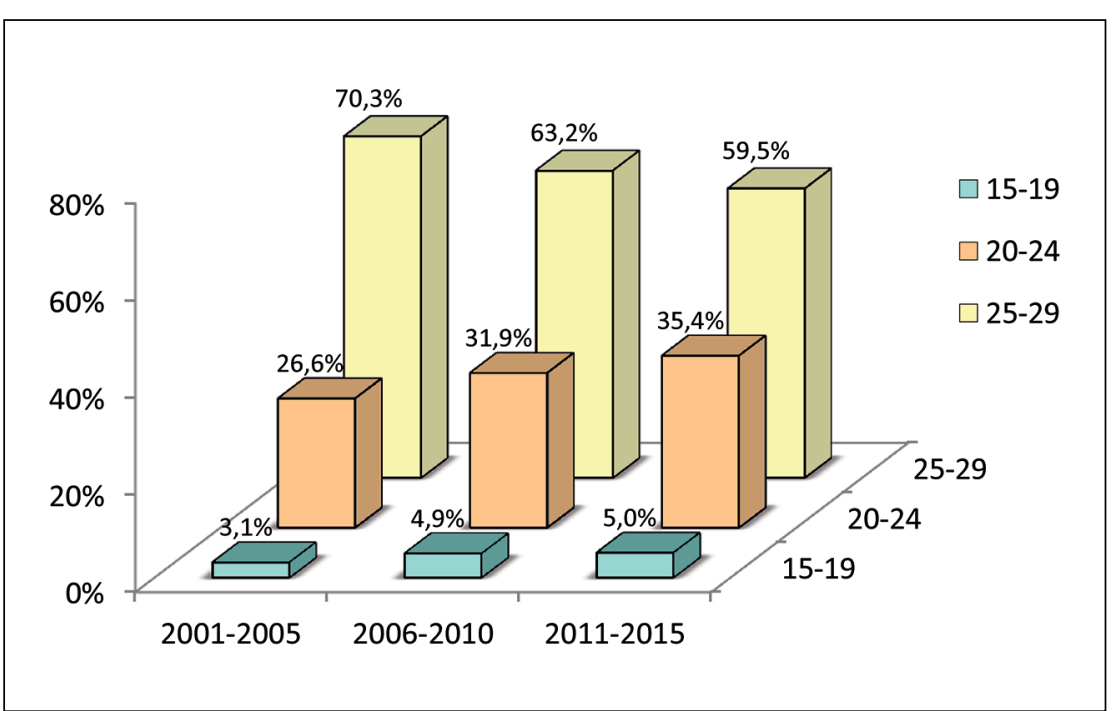

Figura 2. Distribución de edad (15-29 años) de casos que inician TARV de la Cohorte Chilena, por quinquenio. Total de casos quinquenios 2001-2005: 1.225; quinquenio 2006-2010: 1.450; quinquenio 2011-2015: 1.327 . 


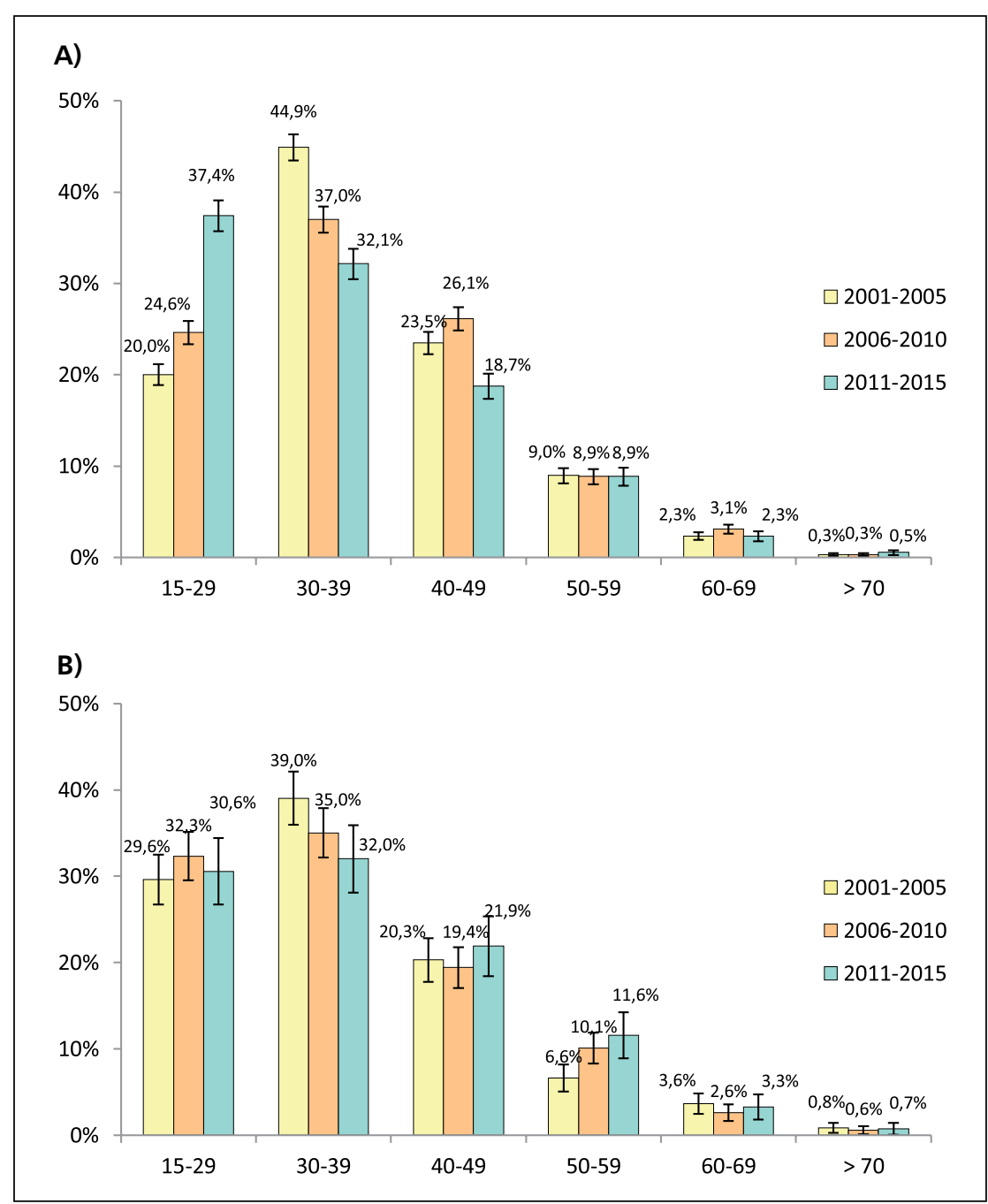

Figura 3. Distribución por edad al inicio de TARV por quinquenio, hombres y mujeres. A) Hombres: Total de casos quinquenios 2001-2005: 4.692; quinquenio 2006-2010: 4.485; quinquenio 2011-2015: 3.096. En las barras se ilustran intervalos de confianza 95\%. B) Mujeres: Total de casos quinquenios 2001-2005: 966; quinquenio 2006-2010: 1.071; quinquenio 2011-2015: 553. En las barras se ilustran intervalos de confianza $95 \%$.

Presentación tardía a cuidados y terapia: características clínicas e inmunológicas al ingreso

En los últimos tres quinquenios se observa que el inicio de TARV con LT CD4 $<200$ céls $/ \mathrm{mm}^{3}$ ha disminuido desde 79,7 a 61,6 y a $42,4 \%$ (Figura 4). Por el contrario, la proporción de pacientes que inicia TARV con rangos de $200-499$ y $>500$ céls $/ \mathrm{mm}^{3}$ ha aumentado sostenidamente. Usando el punto de corte utilizado en numerosas guías clínicas, el inicio de TARV con LT CD4 > 350 céls/ $\mathrm{mm}^{3}$ ha aumentado de 5,8 a 9,8 y a $24,9 \%$ en los tres quinquenios estudiados.

La presentación tardía se asocia también a diagnóstico en etapas clínicas sintomáticas B o C. El diagnóstico en estas etapas ha disminuido desde $70,1 \%$ entre 2001 y 2005 (45,4\% en etapa C) a $33,1 \%$ entre 2011 y 2015 (22,6\% en etapa C) (Figura 5). También observamos que el nivel de CV al inicio de TARV es alto; $42,2 \%$ de los pacientes inicia TARV con $\mathrm{CV}>100.000$ copias $/ \mathrm{mL}$ $(13,8 \%$ con $\mathrm{CV}$ basal $>500.000$ copias $/ \mathrm{mL})$, con una tendencia a disminuir a lo largo de los tres quinquenios (datos no mostrados).

La presentación tardía a TARV presenta diferencias significativas en el análisis por sexo. En el primer quinquenio analizado, el inicio de TARV en etapa $\mathrm{C}$ fue de $33,4 \%$ en mujeres y de $47,8 \%$ en hombres, diferencias que se acortan a 19,4 y $23,1 \%$, respectivamente, en el último quinquenio (Figuras 6a y 6b). La presentación con LT CD4 $<200$ céls $/ \mathrm{mm}^{3}$ tiene un comportamiento similar. La presentación tardía a TARV también aumenta a mayor edad de presentación (datos no mostrados).

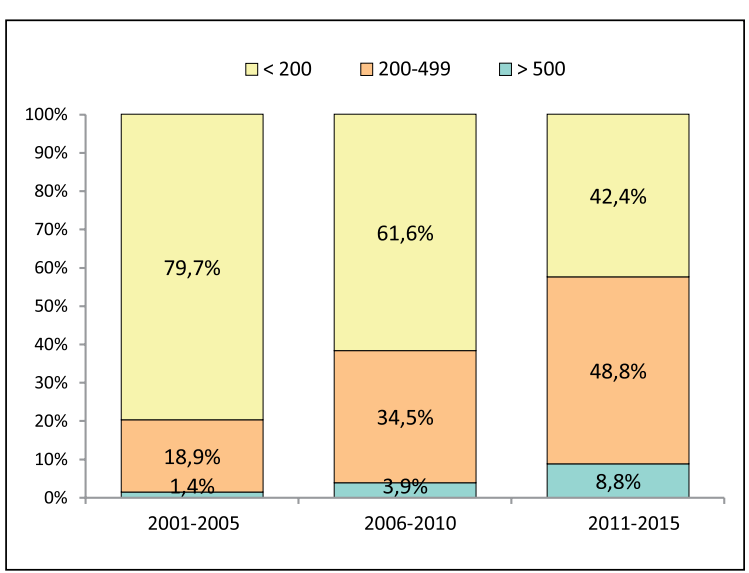

Figura 4. Distribución por etapa inmunológica $\left(\mathrm{LT} \mathrm{CD} 4 / \mathrm{mm}^{3}\right)$ al inicio de TARV, por quinquenio. Total de casos quinquenios 2001-2005: 5.133; quinquenio 2006-2010: 5.176; quinquenio 2011-2015: 3.494.

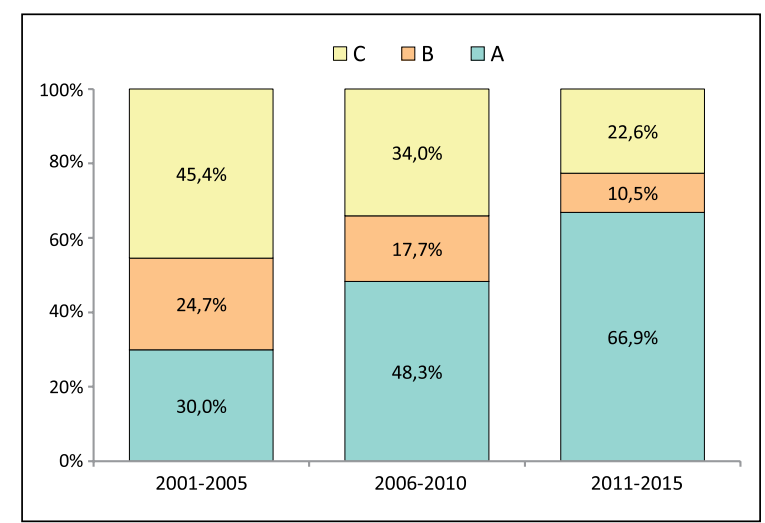

Figura 5. Distribución por etapa clínica CDC al inicio de TARV, por quinquenio. Total de casos quinquenios 2001-2005: 5.268; quinquenio 2006-2010: 5.279; quinquenio 2011-2015: 3.442. 


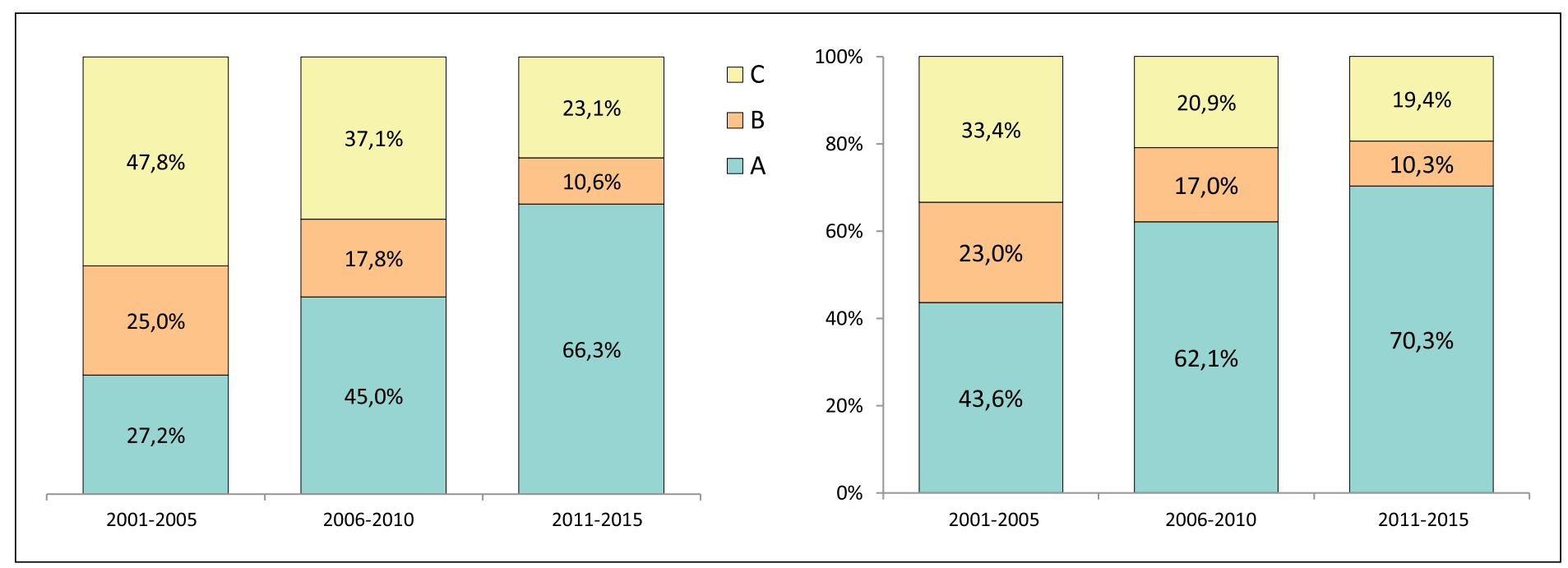

Figura 6. Distribución por etapa clínica CDC al inicio de TARV, por quinquenio, hombres y mujeres. A) Hombres: Total de casos quinquenios 2001-2005: 4.381; quinquenio 2006-2010: 4.269; quinquenio 2011-2015: 2.927. B) Mujeres: Total de casos quinquenios 2001-2005: 887; quinquenio 2006-2010: 1.010; quinquenio 2011-2015: 515.

Dentro de las enfermedades oportunistas más frecuentemente observadas entre los pacientes que se presentan en etapa $\mathrm{C}$ en Chile, se encuentran neumonía por Pneumocystis jiroveci (44,4\%), seguido por síndrome consuntivo $(12,8 \%)$, tuberculosis $(12,4 \%) \mathrm{y}$, con menos frecuencia, sarcoma de Kaposi (SK) y candidiasis esofágica. No se observan cambios significativos en sus proporciones en los tres quinquenios analizados (Tabla 2). Es importante destacar una tendencia a la presentación de enfermedades de etapa $\mathrm{C}$ con recuentos de LT CD4

\begin{tabular}{|c|c|c|c|c|}
\hline Enfermedad oportunista & 2001-2005 & $\begin{array}{c}\text { Quinquenio } \\
2006-2010\end{array}$ & 2011-2015 & Total \\
\hline Neumonía por $P$. jiroveci & $43,2 \%$ & $45,7 \%$ & $45,1 \%$ & $44,4 \%$ \\
\hline Síndrome consuntivo & $14,3 \%$ & $11,6 \%$ & $10,8 \%$ & $12,8 \%$ \\
\hline Tuberculosis & $11,9 \%$ & $12,3 \%$ & $14,2 \%$ & $12,4 \%$ \\
\hline Sarcoma de Kaposi & $8,7 \%$ & $8,6 \%$ & $6,6 \%$ & $8,3 \%$ \\
\hline Candidiasis esofágica & $8,1 \%$ & $7,5 \%$ & $7,9 \%$ & $7,9 \%$ \\
\hline Toxoplasmosis cerebral & $3,8 \%$ & $4,0 \%$ & $4,0 \%$ & $3,9 \%$ \\
\hline Criptococosis meníngea & $2,9 \%$ & $3,0 \%$ & $3,7 \%$ & $3,1 \%$ \\
\hline Linfoma no Hodgkin & $2,0 \%$ & $3,5 \%$ & $2,9 \%$ & $2,7 \%$ \\
\hline Criptosporidiasis crónica & $1,6 \%$ & $0,6 \%$ & $0,3 \%$ & $1,0 \%$ \\
\hline Leucoencefalopatía multifocal & $1,0 \%$ & $0,8 \%$ & $1,3 \%$ & $1,0 \%$ \\
\hline Citomegalovirus no MNI & $0,4 \%$ & $0,6 \%$ & $1,3 \%$ & $0,6 \%$ \\
\hline Encefalopatía por VIH & $0,5 \%$ & $0,4 \%$ & $1,1 \%$ & $0,6 \%$ \\
\hline Úlceras herpéticas crónicas & $0,5 \%$ & $0,5 \%$ & $0,3 \%$ & $0,5 \%$ \\
\hline Micobacteriosis atípica & $0,4 \%$ & $0,7 \%$ & $0,1 \%$ & $0,5 \%$ \\
\hline Cáncer cervico-uterino invasor & $0,3 \%$ & $0,2 \%$ & $0,1 \%$ & $0,2 \%$ \\
\hline Isosporosis crónica & $0,3 \%$ & $0,0 \%$ & $0,0 \%$ & $0,1 \%$ \\
\hline Salmonelosis recurrente & $0,0 \%$ & $0,0 \%$ & $0,3 \%$ & $0,1 \%$ \\
\hline
\end{tabular}




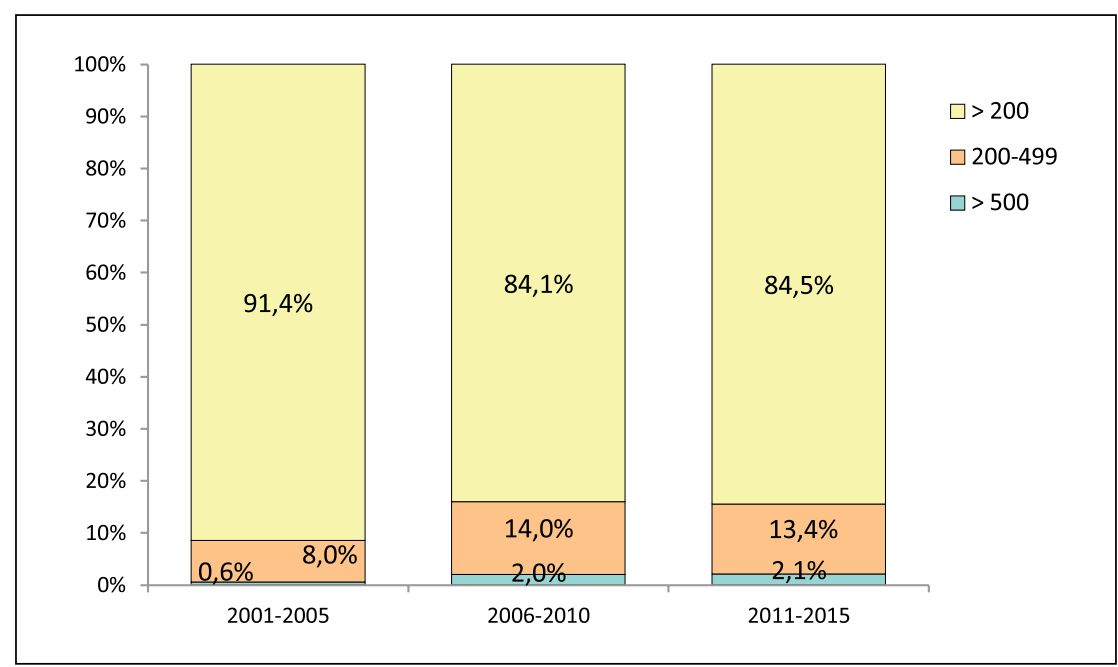

Figura 7. Enfermedades oportunistas de etapa C, según LT CD4. Total de casos quinquenios 2001 2005: 2.311; quinquenio 2006-2010: 1.769; quinquenio 2011-2015: 760.

más altos. En el último quinquenio en Chile, $15,5 \%$ de las enfermedades oportunistas se presenta en pacientes con LT CD4 $>200$ céls $/ \mathrm{mm}^{3}$, especialmente $P$. jiroveci, tuberculosis, sarcoma de Kaposi, síndrome consuntivo, candidiasis esofágica y linfoma no Hodgkin; $2,1 \%$ de las enfermedades oportunistas se presenta en pacientes con LT CD4 >500 céls $/ \mathrm{mm}^{3}$ (Figura 7).

\section{Discusión}

Este estudio representa las características epidemiológicas y clínicas de los pacientes que inician TARV en el sistema público chileno por cuanto incluye pacientes de todos los centros de atención, en forma proporcional a su distribución. Nuestro estudio se refiere sólo a los pacientes que inician TARV; sin embargo, aproximadamente $90 \%$ de las personas con diagnóstico de infección por VIH en Chile, se encuentra recibiendo TARV, por lo que las características epidemiológicas y clínicas aquí descritas podrían ser extrapolables al total de pacientes. En Chile, la epidemia de infección por VIH es de tipo concentrada en poblaciones clave como la de hombres que tienen sexo con hombres (HSH) y los informes epidemiológicos del Ministerio de Salud señalan que 16,3\% del total de casos VIH/SIDA reportados en el país entre 1984 y 2013 corresponde a mujeres ${ }^{7}$, resultados concordantes con los nuestros de 17,4\%. El Ministerio de Salud describe una tendencia leve al aumento en la proporción de mujeres infectadas por VIH en los últimos años, lo que no se ve reflejado en el análisis por quinquenios de nuestro estudio.

Las cifras nacionales oficiales dan cuenta de la mayor prevalencia de casos entre 30 y 49 años de edad, en con- cordancia con lo reportado en este estudio; no obstante, se notifica un aumento significativo en el número de nuevos casos entre 15 y 29 años ${ }^{7}$. Nuestros resultados también reflejan esta situación mostrando un aumento en la proporción de pacientes entre 15 y 29 años que inician TARV, desde 21,7 a $36,4 \%$ en los tres quinquenios reportados (Figura 1), en concordancia con reportes latinoamericanos que dan cuenta de esta situación ${ }^{8}$. Este aumento en la proporción de casos ocurre principalmente entre 15 y 24 años, lo que podría obedecer a un aumento en la transmisión de la infección por VIH entre adolescentes y adultos jóvenes, o bien, a una mayor detección de casos por focalización de testeo en este grupo de edad. Sin embargo, la ausencia de campañas orientadas a la promoción del testeo y las estadísticas oficiales del Ministerio de Salud que muestran que la pesquisa de VIH continúa realizándose mayoritariamente en el contexto de embarazo y donación de sangre, nos lleva a concluir que hay un aumento en la transmisión de VIH entre adolescentes y adultos jóvenes en el país.

En nuestro estudio observamos que $12,1 \%$ de la población inició TARV con 50 y más años, con un sensible aumento en las mujeres. Estos resultados señalan la necesidad de contar con estudios comportamentales en mayores de 50 años, en especial en mujeres, además del diseño de políticas de prevención que incluyan este grupo etario. Esta cifra es concordante con lo observado en otros estudios de Latinoamérica, donde se reporta $15,2 \%$ de personas que inician control sobre los 50 años $^{8}$ y representa un desafío importante para la atención de las personas infectadas por VIH, que se suma al envejecimiento de la población infectada a edades más jóvenes. En nuestro estudio se confirma la prevalencia, al ingreso a control, de co-morbilidades tales como hipertensión arterial, diabetes mellitus y dislipidemia, en pacientes mayores de 50 años, lo que se asocia a un mayor riesgo cardiovascular en personas infectadas por VIH. No obstante, la prevalencia de estas tres patologías observada en los pacientes de más de 50 años que inician TARV en la cohorte, es inferior a la observada en estudios realizados en población general del país. La mediana de edad de los pacientes infectados por VIH va en aumento. En la Cohorte ATHENA, 28\% de los pacientes son mayores de 50 años y la edad promedio es de 43,9 años, estimándose que los mayores de 50 años podrían llegar a $73 \%$ en $2030^{2}$; esta cifra es algo menor en Latinoamérica con $21,5 \%$ de los pacientes en control mayor de 50 años (análisis de 57.290 pacientes del Taller Latinoamericano de $\left.\mathrm{VIH}^{10}\right)$.

La Organización Mundial de la Salud (OMS) y ONUSIDA han planteado las ambiciosas metas 90-90-90 para 2020 y 95-95-95 para 2030 (95\% de diagnóstico entre las personas infectadas por VIH, vincular a atención e iniciar terapia en $95 \%$ de ellos y lograr $95 \%$ de CV suprimida a menos de 1.000 copias $/ \mathrm{ml}$ en $95 \%$ de los 
tratados) en el contexto de la Estrategia 2.0 y una respuesta acelerada que pretende terminar con la epidemia de SIDA ${ }^{11}$. Para alcanzar las metas 90-90-90 al 2020, se deben enfrentar brechas importantes, especialmente en diagnóstico, que a nivel mundial alcanza actualmente sólo 53\% de las personas infectadas y en Chile ha sido estimado en $73 \%$ por OMS y ONUSIDA y $53 \%$ del total de infectados actualmente recibiendo TARV. Sin embargo, todos los análisis de sub-diagnóstico se basan en estimaciones de población infectada comparados con las personas en control; esta tasa podría ser mayor. Un parámetro complementario para estimar la falta de diagnóstico es la medición de la presentación tardía a control, definida para estos fines como el primer recuento de LT CD4 $<200$ céls $/ \mathrm{mm}^{3}$, cuya reducción a $10 \%$ constituye la "cuarta meta" de OMS. La tasa de presentación a TARV con LT CD4 $<200$ céls $/ \mathrm{mm}^{3}$ en Latinoamérica, para los años 2013-2014, es de 36\%, en base a un estudio en 22 centros de 9 países ${ }^{12}$, y aún mayor en reportes de años anteriores de la red CCASAnet, que además demuestran que mayoritariamente el inicio tardío de TARV obedece a diagnóstico tardío y, en menor medida, a retraso en el inicio de TARV ${ }^{13}$. Nuestro estudio ratifica estos hallazgos demostrando una reducción en el inicio de TARV con LT CD4 < 200 céls $/ \mathrm{mm}^{3}$, desde 79,7 a $42,4 \%$ en los años recientes, lo que aún es muy distante de la meta OMS. Además contribuye a enfatizar la necesidad de un diagnóstico más oportuno de la infección por VIH y la urgencia de concentrar los esfuerzos en abolir las barreras al diagnóstico si se pretende avanzar en las metas 90-90-90. El problema de la presentación tardía es más frecuente en hombres que mujeres (38,2 vs 34,7\%) y a edades mayores por lo que, nuevamente, se deben diseñar políticas específicas para esta población, que incluyan mayor oferta de testeo.

La presentación a TARV con LT CD4 < 200 céls $/ \mathrm{mm}^{3}$ se asocia a una elevada presentación de enfermedades oportunistas previas o al momento del inicio de la TARV y a CV basales elevadas. Estos eventos muestran, al igual que la presentación con LT CD4 bajos, el mismo comportamiento por sexo y edad y la misma tendencia a mejorar pero en forma insuficiente en los últimos años. Nuestro estudio no muestra cambios significativos en el tiempo en el tipo de enfermedades oportunistas que presentan los pacientes infectados con VIH en Chile. No obstante, en concordancia con los estudios START y Temprano, que demostraron en pacientes con diagnóstico temprano la presentación de enfermedades oportunistas con más de 500 LT CD $4^{14,15}$, nosotros también observamos una tendencia a la presentación de enfermedades de etapa $\mathrm{C}$ con recuentos de LT CD4 más altos en el último quinquenio, en que una mayor proporción de pacientes se presentó con menor deterioro inmunológico. Estos hallazgos enfatizan la necesidad de modificar la guía clínica de TARV chilena para ofrecer TARV a todos los pacientes con infección por VIH, de manera independiente de su recuento de LT CD4, como lo recomienda la mayoría de las guías clínicas internacionales y de la $\mathrm{OMS}^{16-18}$.

Una limitación importante de este estudio corresponde a que los datos del quinquenio 2011-2015 aún se encuentran en procesos de extracción y validación por lo que no pudieron ser incluidos en su totalidad para el análisis actual. Esto limitó la posibilidad de construir tasas y riesgos poblacionales. No obstante, cuando fue pertinente, analizamos las tasas de los primeros dos quinquenios, corroborando las tendencias en sus dos primeros puntos. Por esta razón, es necesario observar de manera prudente las tendencias descritas. Sin embargo, la distribución de la información recolectada para el último quinquenio según centro (Tabla 1) mantiene, en la mayoría de los casos, similar proporcionalidad que la observada en los quinquenios anteriores. Otra limitación corresponde a los datos faltantes en algunas de las variables de interés. No obstante, como se observa en los valores totales de cada tabla o figura de sexo, edad, LT CD4 y clasificación CDC, éstos son siempre inferiores a $10 \%$.

Estas consideraciones no limitan la relevancia de los datos presentados. El presente reporte entrega información clave para comprender las características y evolución de la epidemia en Chile en los últimos 15 años. Por una parte, hay una tendencia a la presentación de la infección a edades más jóvenes en hombres mientras que en mujeres un grupo de preocupación creciente corresponde a las mayores de 50 años. Además hemos observado que, pese a los avances en acceso a tratamiento que han permitido reducir la presentación a tardía a terapia, aún persisten importantes desafíos para alcanzar un inicio de TARV más oportuno, en concordancia con las metas 90-90-90 de OMS.

\section{Resumen}

Introducción: La Cohorte Chilena de SIDA es la más antigua y extensa de Latinoamérica y una de las más numerosas y con mayor tiempo de seguimiento a nivel internacional. Registra información de 14.873 pacientes de los aproximadamente 22.000 en TARV en el sistema público y sus resultados han permitido conocer la realidad nacional y han contribuido a la adopción de políticas públicas. El objetivo de este estudio es describir las características demográficas, clínicas e inmunológicas de los pacientes que han comenzado TARV en Chile y su evolución en los últimos 15 años. Pacientes y Métodos: Los casos fueron estratificados por quinquenios: 20012005, 2006-2010 y 2011-2015. El análisis de los datos incluyó el cálculo de proporciones, sus respectivos intervalos de confianza $95 \%$ y se aplicó test de $\chi^{2}$ para análisis 
de significación. Resultados: El 17,4\% de los casos que inician TARV corresponde a mujeres y la proporción se ha mantenido relativamente constante. Por edad, destaca el grupo de adultos entre 30 y 39 años aunque el estrato de 15-29 años evidencia un importante aumento desde 21,7 a $36,4 \%$ en $2011-2015$, especialmente en hombres. Un $12,1 \%$ del total de los inicios de TARV son mayores de 50 años con una tendencia estable en el tiempo; sin embargo, las mujeres mayores de 50 años han aumentado de 11,0 a 15,6\%. El inicio de TARV con LT CD4 menor de 200 céls $/ \mathrm{mm}^{3}$ ha disminuido desde 79,7 a $42,4 \%$ y en etapa C desde 45,4 a 22,6\%. La presentación tardía a TARV es mayor en hombres pero esta diferencia se ha reducido en el último quinquenio. Neumonía por Pneumocystis jiroveci, síndrome consuntivo, tuberculosis, sarcoma de Kaposi y candidiasis esofágica son las enfermedades oportunistas más frecuentes sin cambios significativos en los tres quinquenios analizados. En el último quinquenio, $15,5 \%$ de las enfermedades oportunistas se presenta en pacientes con LT CD4 >200 céls/mm³. Discusión: Pese a las limitaciones de los estudios observacionales el presente reporte describe las características y evolución de la epidemia en Chile en los últimos 15 años. La infección se presenta a edades más jóvenes en hombres, mientras que en mujeres hay un aumento en mayores de 50 años. Pese a los avances en acceso a tratamiento que han permitido reducir la presentación a tardía a terapia, aún persisten importantes desafíos para alcanzar un inicio de TARV más oportuno, en concordancia con las metas 90-90-90 de OMS.

\section{Referencias bibliográficas}

1.- Lazcano-Ponce E, Fernández E, SalazarMartínez E, Hernández-Avila M. Estudios de cohorte. Metodología, sesgos y aplicación. Rev Salud Pública México 2000; 42 (3): 230-41.

2.- Smit M, Brinkman K, Geerlings S, Smit C, Thyagarajan K, Sighem AV, et al. ATHENA observational cohort. Future challenges for clinical care of an ageing population infected with HIV: a modeling study. Lancet Infect Dis. 2015; 15 (7): 810-8. doi: 10.1016/S14733099(15)00056-0. Epub 2015 Jun 9.

3.- Lundgren J, Phillips A, Vella S, Katlama C, Ledergerber B, Johnson A, et al. Regional differences in use of antiretroviral agents and primary prophylaxis in 3122 European HIVinfected patients. EuroSIDA Study Group. J Acquir Immune Defic Syndr Hum Retrovirol 1997; 16 (3): 153-60.

4.- Gange S, Kitahata M, Saag M, Bangsberg M, Bosch R, Brooks J, et al. Cohort Profile: The North American AIDS Cohort Collaboration on Research and Design (NA-ACCORD). Int J Epidemiol. 2007; 36 (2): 294. doi:10.1093/ije/ dyl286

5.- Wolff M, Beltrán C, Vásquez $\mathrm{P}$, Ayala $\mathrm{M}$, Valenzuela M, Berríos G, et al. The Chilean AIDS Cohort. A model for evaluating the impact of an expanded access program to antiretroviral therapy in a middle-income country-Organization and preliminary results. J Acquir Immune Defic Syndr 2005; 40 (5): 551-7.

6.- Wolff M, Cortés C, Shepherd B, Beltrán C for the Chilean AIDS Cohort Study Group.
Long-term outcomes of a national expanded access program to antiretroviral therapy: The Chilean AIDS Cohort. J Acquir Immune Defic Syndr 2010; 55 (3): 368-74.

7.- Departamento de Epidemiología División de Planificación Sanitaria Ministerio de Salud de Chile. Informe nacional parte I Evolución VIH/SIDA, Chile 1984-2013. Acceso en mayo 2016 en: http://epi.minsal.cl/wp-content/ uploads/2016/02/InformePais_1984-2013_vih_ sida.pdf

8.- Gallo C, López O, Chahin C, Marincovich B, Zitko P, Beltrán C on behalf of The Latinamerican Workshop Study Group. Significant increase in new HIV infections among young adults in Latin America. HIV and Hepatitis in Americas; Mexico 2016. P037

9.- Ministerio de Salud de Chile - P. U. Católica de Chile. Encuesta Nacional de Salud 2009-2010. Acceso en mayo 2016 en: http://web.minsal.cl/portal/url/item/ bcb03d7bc28b64dfe040010165012d23.pdf

10.- Beltrán C, Sued O, Celi AP, Castillo P, Thormann M, López O, et al on behalf of The Latinamerican Workshop Study Group. Ageing in Latin America; results of a large collaborative study group. J Int AIDS Soc 2015; 18 (Sup 2): S25-6.

11.- UNAIDS. Fast track; ending the AIDS epidemic by 2030 . Acceso en mayo de 2016 en: http:// www.unaids.org/en/resources/documents/2014/ JC2686_WAD2014report

12.- Celi AP, Greco M, Martínez E, Vargas C, Belaunzaran F, Mejía F, on behalf of The Latinamerican Workshop Study Group. Presentation to care with advanced HIV disease is still a problem in Latin America. HIV and Hepatitis in Americas; Mexico 2016. P038.

13.- Crabtree-Ramírez B, Caro-Vega Y, Shepherd B, Wehbe F, Cesar C, Cortés C, et al on behalf of the CCASAnet Team. Cross-sectional analysis of late HAART initiation in Latin America and the Caribbean: Late testers and late presenters. PLoS One May 2011; 6 (5) e20272.

14.- The INSIGHT START Study Group. Initiation of antiretroviral therapy in early asymptomatic HIV infection. N Engl J Med. 2015; 373 (9): 795-807. DOI: 10.1056/NEJMoa1506816. Epub 2015 Jul 20.

15.- The TEMPRANO ANRS 12136 Study Group. A trial of early antiretrovirals and isoniazid preventive therapy in Africa. N Engl J Med 2015, N Engl J Med 2015; 373 (9): 808-22. DOI: 10.1056/NEJMoa1507198. Epub 2015 Jul 20.

16.- World Health Organization. Consolidated Guidelines on the Use of Antiretroviral Drugs for Treating and Preventing HIV Infection. November 2015. Acceso en mayo de 2016 en: http://www.who.int/hiv/pub/arv/policy-briefarv-2015/en/

17.- DHHS Panel on Antiretroviral Guidelines for Adults and Adolescents. Guidelines for the Use of Antiretroviral Agents in HIV-1-Infected Adults and Adolescents (Acceso en julio de 2016 en: http://aidsinfo.nih. gov/guidelines)

18.- European AIDS Clinical Society. EACS Guidelines 8.0 October 2015. Acceso en junio de 2016 en: http://www.eacsociety.org/ guidelines/eacs-guidelines/eacs-guidelines.html 